\title{
GUEST EDITOR'S LETTER
}

\author{
V. A. YURKO
}

This issue is devoted to inverse problems of spectral analysis for ordinary differential operators. Inverse spectral problems consist in recovering operators from their spectral characteristics. Such problems often appear in mathematics, mechanics, physics, electronics, geophysics, meteorology and other branches of natural sciences. Inverse problems also play an important role in solving nonlinear evolution equations in mathematical physics. Interest in this subject has been increasing permanently because of the appearance of new important applications, and nowadays the inverse problem theory develops intensively all over the world. The goal of this issue is to present latest results in the inverse spectral problem theory for ordinary differential operators and discuss their possible applications. 